\section{Request for brightness matching data and mathematical color vision models}

\section{JO ANN S. KINNEY \\ U.S. Representative, CIE Committee TC 1.4}

The principles of light measurement as a true visual quantity are discussed in a recent technical report prepared by CIE (Note 1). The great achievements of the CIE in defining the photopic and scotopic standard observers were noted. Experience with these observers has shown that, while the scotopic standard luminous efficiency function $\left(V^{\prime} \lambda\right)$ is satisfactory, the photopic function $V(\lambda)$ has some drawbacks. $V(\lambda)$ serves adequately enough for assessing many continuous spectral broad-band light sources, according to the equation:

$$
L_{v}=K_{m} \int_{360}^{830} L_{e \lambda} V(\lambda) d \lambda \text {, }
$$

where $\mathrm{L}_{\mathrm{v}}=$ luminance in $\mathrm{cd} / \mathrm{m}^{2}, \mathrm{~L}_{\mathrm{e} \lambda}=$ spectral concentration of radiance in $\mathrm{Wm}^{-2} \mathrm{sr}^{-1}, \mathrm{~V}(\lambda)=$ CIE photopic luminous efficiency function, and $\mathrm{K}_{\mathrm{m}}=$ maximum spectral luminous efficacy $\left(683 \mathrm{lmW} \mathrm{m}^{-1}\right)$.

This is adequate because, with these types of sources, equally bright lights are also approximately equal in luminance. However, as noted in the aforementioned CIE technical report (Note 1) and in a number of papers referenced in this report, lights equal in luminance are not always equally bright. This discrepancy is most obvious with narrow-band sources, whether restricted to one wavelength region such as line sources or leds or with achromatic sources with irregular spds, such as mercury vapor lamps.

It is explicitly noted in the CIE report (Note 1) that "This report is recommended for future study and is not an officially agreed CIE recommendation." Any recommendations offered in the report are advisory only.

The purpose of this communication is to propose a timetable for the future plans of CIE TC 1.4 so that all workers may know the probable form of future official recommendations. Such recommendations cannot be made now because the necessary information is not complete. Committee TC 1.4 strongly recommends the gathering of relevant data in order that firm recommendations can be made at the quadrennial meeting of the CIE in 1983.

The recommendations will use the present CIE standard observers, $\bar{x}, \bar{y}, \bar{z}, \bar{x}_{10}, \bar{y}_{10}, \bar{z}_{10}, V^{\prime}(\lambda)$, in conjunction with mathematical models of the human visual system to convert standard light and colorimetric measures to visually meaningful light measurements. Before that time, preferably at the 1979 quadrennial meeting of the CIE in Kyoto, Japan, from existing data, the average spectral sensitivities of human observers will be defined under the following conditions:

Heterochromatic brightness matching to standards of $570 \mathrm{~nm}$, Illustration A and Illustration D65: ${ }^{1}$ (a) $10^{\circ}$ field, (b) $2^{\circ}$ field, and (c) point sources.

These data should be obtained for an adult population aged 20 to 50 years. In addition, data on both younger and older individuals would be very useful.

At the 1983 CIE Meeting, methods to convert standard photometric and colorimetric data to light measures which are a "true visual quantity" will be recommended. In particular, it is intended:

(1) To express mesopic luminances which are derived from $V(\lambda)$ and $V^{\prime}(\lambda)$. These derived luminances should predict the function published by Kinney (1964) and Walters and Wright (1943).

(2) To express photopic brightness functions derived from $\bar{x}, \bar{y}, \bar{z}, \bar{x}_{10}, \bar{y}_{10}, \bar{z}_{10}$. These derivations would be accomplished with the aid of a mathematical model of vision designed for this purpose. Such models are discussed in the CIE technical report (Note 1). While several such models are reviewed therein, we are as yet not prepared to recommend any one as suitable for these derivations. Thus, we encourage visual scientists to incorporate such derivations in their vision models. The vision model must predict brightness matching sensitivity function at clearly suprathreshold photopic levels. Ideally, the model should handle $2^{\circ}$, $10^{\circ}$, and point source fields. It may be modified for young and old populations.

\section{REFERENCE NOTE}

1. CIE. Light as a true visual quantity: Principles of measurement. Technical report, CIE Technical Committee 1.4, "Vision," 1978.

\section{REFERENCES}

Kinney, J. A. S. The effect of field size and position on mesopic spectral sensitivity. Journal of the Optical Society of America, 1964, 54, 671-677.

WALters, H. V., \& WRIGHT, W. D. The spectral sensitivity of the fovea and extrafovea in the Purkinje range. Proceedings of the Royal Society (London) Section B, 1943, 131, 340-361.

\section{NOTE}

$1.570 \mathrm{~nm}$ is recommended as a standard because (1) it is reasonably easy to obtain and (2) it is at this wavelength that spectral luminosity functions obtained by brightness matching and flicker photometry differ the least on an absolute scale.

CIE illuminant $A$ is obtained by operating a tungsten lamp at $2,854 \mathrm{~K}$.

CIE illuminant D65 can be approximated by filtering illuminant A with Kodak Wratten Filter 78AA.

(Received for publication and accepted May 8, 1978.) 